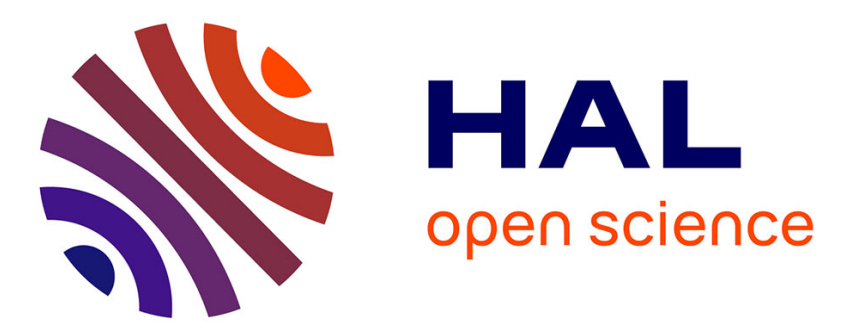

\title{
Model Based Compensation for Low Mass Objects Haptic Manipulation in Virtual Environments
}

Simon Hilt, Charles Pontonnier, Georges Dumont

\section{To cite this version:}

Simon Hilt, Charles Pontonnier, Georges Dumont. Model Based Compensation for Low Mass Objects Haptic Manipulation in Virtual Environments. Proceedings of EuroVR conference 2017, Dec 2017, Laval, France. pp.87-101, 10.1007/978-3-319-72323-5_6 . hal-01626147

\section{HAL Id: hal-01626147 https://hal.inria.fr/hal-01626147}

Submitted on 30 Oct 2017

HAL is a multi-disciplinary open access archive for the deposit and dissemination of scientific research documents, whether they are published or not. The documents may come from teaching and research institutions in France or abroad, or from public or private research centers.
L'archive ouverte pluridisciplinaire HAL, est destinée au dépôt et à la diffusion de documents scientifiques de niveau recherche, publiés ou non, émanant des établissements d'enseignement et de recherche français ou étrangers, des laboratoires publics ou privés. 


\title{
Model Based Compensation for Low Mass Objects Haptic Manipulation in Virtual Environments
}

\author{
Simon Hilt ${ }^{1,2}$, Charles Pontonnier ${ }^{1,2,3}$ and Georges Dumont ${ }^{1,2}$ \\ 1 IRISA/INRIA/M2S MimeTic \\ 2 École Normale Suprieure de Rennes \\ 3 Écoles de Saint-Cyr Coetquidan
}

\begin{abstract}
This paper aims at presenting a model based compensation command law developed in order to enhance the transparency of haptic interfaces for low mass objects manipulations in virtual environments. After presenting the context and related work of the study, the model based compensation command law is developed and implemented on a 6-dofs haptic interface. Uncertainties of the model (friction coefficients) are then tuned thanks to an experimental protocol enabling a subjective comparison between real and virtual manipulations of a low mass object. Results of this experimentation are presented and discussed. The compensation of friction on the first and second axes of the haptic interface showed significant improvement of both realism and perceived load. A short conclusion opens perspectives to this work at the end of the paper. Such a work has the potential to enhance the fidelity of interaction for ergonomics applicaitions. Keywords: Haptics, Dynamics, Fidelity, Ergonomics, Control.
\end{abstract}

\section{Introduction}

Virtual Reality (VR) is emerging as a powerful tool for ergonomics, rehabilitation or sports applications. In all of these domains, it is mandatory to guarantee that the results and conclusion made from a virtual setup are transferable to the real world. In the ergonomics case, it is clear that the key point to make virtual environments usable in early design stage of workstations (prototyping shapes, locations, tools of a specified industrial workstation from a digital mock up (DMU)) is to be sure that any biomechanical impact evaluated during a virtual reality ergonomics design session has any reliability with the one observed for real.

In such a setup, the user is immersed in a virtual environment (VE) simulating his real working environment (RE), and he is asked to perform tasks in interaction with the $\mathrm{VE}$ corresponding to ones he would perform in RE. Thus, its activity is monitored and analyzed by external actors [1] such as an ergonomist. In cases involving particularly low level of force exertion, the interaction can be ensured thanks to a 3Djoystick [2-6]. However, tasks requiring for a moderate or high level of 
force to be fulfilled cannot be properly simulated with such a device. Indeed, to ensure a high fidelity level for such cases, it is necessary to propose an interaction enabling forces exertion between the user and the virtual environment $[7,8]$. Haptic devices have the ability to ensure this realistic interaction since they provide a force feedback to the user when this user interacts with a virtual object. Such a device has to exhibit a good transparency, i.e. the haptic interface should transmit the virtual environment impedance to the user without distortion, and stability $[9$, 10] to be used this way.

The current paper aims at proposing a command law based on dynamic compensation - Model Based Compensation - to control a 6 degrees of freedom (dofs) haptic interface (HI) as a transparent and stable interface between a user and a VE. Particularly, the effect of compensation of viscous friction effects on the realism and the perception of the task by the users is assessed through an experimental protocol. The use case chosen to illustrate the relevance of such an algorithm is low mass object manipulations, in which the inertia and friction of the $\mathrm{HI}$ is not negligible with regard to the load to manipulate. The novelty of the contribution lies in the implementation of such control law on a 6 -dofs and high-torque haptic interface and the experimental assessment of the viscous friction compensation effects on the interaction fidelity.

\section{Related Work}

\subsection{Qualifying the haptic interaction in VE}

Classically, the haptic interaction between a user and a VE can be considered as presented in Fig. 1. The user applies a force $F_{r}$ on the interface's handle enabling its motion. This motion is measured through motor encoders and the current position of the interface $X_{v}$ is transmitted to the physics simulation. This one will compute a force $F_{v}$ corresponding to the virtual object motion with respect to physical laws (Newton laws, contact models, ...) and will send it to the HI to generate the corresponding force feedback thanks to the actuators.

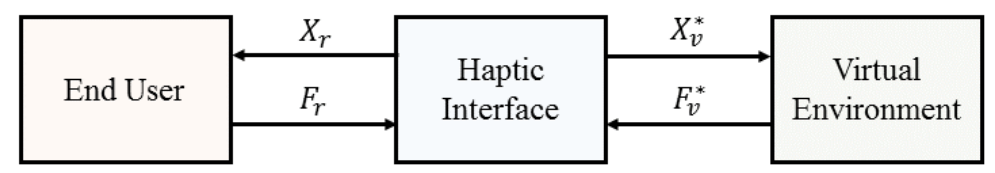

Fig. 1. An overview of the interaction between the user, the HI and the VE.

Such an interaction will be a more or less fair view of a real interaction between a user and an object. The fidelity of the interaction can be characterized through two main components: 
- the stability of the system (in Lyapunov terms [11]);

- the transparency of the system, i.e. its capacity to render the forces arising from the interaction between the object and the user.

These components are affected by several factors, as it has been stated in $[12]$ :

- the inertia and viscous friction of the interface, whose actions pollute the actuators ones;

- the actuators dynamics, adding a delay between the command and the output torque;

- the analogical (human interaction) - numerical (virtual environment) conversion adding delays due to time sampling and conversion schemes, and adding quantification errors;

- the current and position sensors, adding uncertainties to the measured variables.

Several researchers proposed more or less sophisticated methods to limit the influence of these factors on both stability and transparency, as we present it in the next section.

\subsection{Haptic control methods associated to transparency and stability features}

Studies in the literature focus on either the increasing of the stability range or on the improvement of transparency. In order to ensure stability, most methods propose to ensure the passivity of the interaction, i.e. the energy transmitted by the user to the HI must always be greater than the initial stored energy $E_{0}[13]$ :

$$
\int_{0}^{t} F_{r}(\tau) \dot{X}_{r}(\tau) \mathrm{d} \tau>-E_{0}, \forall t>0, \text { admissible } F_{r}(t)
$$

A system that does not satisfy (1) is said to be "active" and unstable vibration starts. Assuming that the transmission delays and the electrical time constant are negligible and that only viscous friction and inertia act on the system, Colgate et al. [13] establish for a 1-dof HI a condition between the physical viscous friction $b$, the sampling time $T$ and the impedance $H(z)$ of the virtual scene, i.e. the ratio between the calculated force and the velocity set-point of the simulation, to ensure passivity:

$$
b>\frac{T}{2} \frac{1}{1-\cos (\omega t)} \operatorname{Re}\left[\left(1-e^{-j \omega T}\right) H\left(e^{j \omega T}\right)\right] \text { for } 0 \leq \omega \leq \frac{\pi}{T}
$$

If the scene represents the contact with a wall of stiffness $K$ and damping $B$, (2) becomes:

$$
b>\frac{K T}{2}+B
$$

According to (2) and (3), instabilities arise for high impedance of the simulation, i.e. when contact with a rigid wall is simulated. Due to technical limitations, the sampling time can not be decreased indefinitely, therefore to increase the stable range of impedance, damping of the HI has to be be increased, at the expense of transparency. Consequently, 
transparency for low impedances and stability for high impedances can't be obtained simultaneously without a control algorithm.

Z-Width plot [14] is used to evaluate the range of impedance that can be transmitted through a HI. On this plot, the zone of passivity or stability is delimited in the plane of damping and stiffness.

Various techniques for ensuring stability have been proposed, relying for the most part on the dissipation of energy either in the haptic interface or in the simulation.

Colgate et al. proposed three methods to increase the damping of the HI [14-16], in order to extend the Z-Width. the addition of an active physical damping to the motor rotor was proposed in order to simulate larger virtual impedances. A frequency-dependent passive electrical damping was suggested. This method consists in a resistance and a capacitor in parallel with the motor. Finally, an active electrical damping proportional to the speed of rotation of the rotor was proposed.

Other methods for increasing the "Z-width" based on the artificial modification of the signals between the HI and the simulation have been proposed. Ryu et al. proposed a time-domain passivity algorithm [17-19]. A passivity observer verifies that the condition of passivity is assured, if not, the passivity controller modifies the control signal of the haptic interface in order to dissipate the excess energy. This ensure the passivity of the interaction, however the signal is modified between the simulation and the haptic interface, at the expense of transparency. Moreover a high sampling frequency is needed. More details of this method is presented in the section 3. An analog input shaper (AIS) [20] is proposed by Lim et al. which performs as a nonlinear low-pass filter that dissipates excess energy. Adjusting output limit (AOL) is another method to ensure stability [21]. Maximum force is tuned to avoid instabilities, but this method can't be used to transmit the contact with stiff wall.

All of these studies improve the " $\mathrm{Z}$ width", but the transparency was never considered. Some methods added to a stability algorithm have been proposed to improve the transparency of the interaction. Franken et al. proposed a two-layers control algorithm [22,23], the first layer guarantees the stability of the interaction and the second layer improves the transparency. Linear lead-lag [24] and adaptive compensation [25] were proposed to improve transparency. Closed loop and Model based compensation are commonly implemented to improve transparency $[26$, 27]. Model based compensation, which requires to identify perfectly the mechanical behavior, is developed in detail in the next section. Recently Baser et al. proposed two methods $[28,29,9]$, the first one is a Torque compensator based on motor current (TCBMC), consisting in adapting the control torque of the haptic interface with regard to the actuator torque. The second one, which extend the TCBMC method, is a hybrid control algorithm by adding an active actuator, which generates a torque 
on the motor shaft.

Most of these techniques have been validated from a system control point of view for a specifically designed 1-dof HI. However, the current paper aims at presenting a model based compensation for HI exhibiting 6 degrees of freedom and and its capacity to enhance transparency, stability and therefore fidelity of the interaction for low mass object manipulations. Particularly, the method is of interest for low mass object manipulations, since the mass to manipulate develop comparable forces as the proper inertia and friction effects of the HI. In the following section, the model based compensation method implemented and the subjective assessment of the effect of the friction compensation for low mass object manipulation are presented.

\section{Materials and Methods}

\subsection{Model Based Compensation Algorithm}

The following section presents the model based compensation algorithm. The algorithm has been implemented in an experimental setup developed with Unity $\AA 5.5 .2$ and its physics engine for the virtual environment, and the Haption SA Virtuose ${ }^{\mathrm{TM}}$ 6D-35-45 and its API for the haptic interface.

HI control scheme The Haption SA Virtuose ${ }^{\mathrm{TM}}$ 6D-35-45 is a haptic interface enabling high force and torque feedback in a volume close from the human arm one. Its internal control -as shown in Fig. 2 - can be summarized as follows: A control loop is implemented on servomotors to control the accuracy and the dynamics of the force feedback. The link between cartesian and joint spaces is made thanks to the jacobian matrix of the HI. The HI communicates with the virtual environment through an ethernet network.

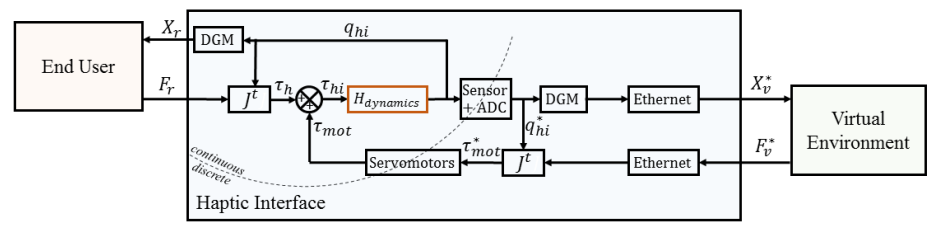

Fig. 2. HI control details.

VE control scheme The object position in the VE is driven by newton laws simulated by a physics engine. In order to enable a realistic 
motion of the object, a control scheme has been developed, as shown in Fig. 3.

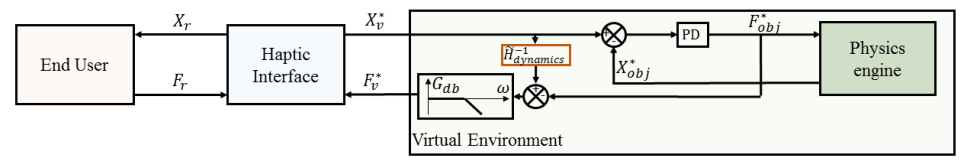

Fig. 3. Virtual environment control details.

Since the physics engine expects forces to be applied to the object in order to make it move (direct dynamics), a virtual proportional-derivative controller has been implemented. The gains of the controller are chosen in order to make the system stiff enough with a reasonable overshoot. Gains have been normalized with regard to the mass of the virtual object to move. Gravity is applied outside of this control scheme in order to avoid brutal changes of the command.

The force applied to the object is also applied to the HI actuators. However, in order to minimize the effects of the HI dynamics, a model based compensation is added and the sum of both actions is low pass filtered before being send to the HI.The model based compensation is presented in the next section.

Model based compensation In order to minimize the dynamics effect associated to inertia and friction, we developed a dynamic model of the HI to generate a compensation force $F_{\text {comp }}^{*}$. As shown in Fig. 4, the haptic interface can be modeled as a 6-dofs kinematical chain with associated masses and inertias obtained from the digital mock-up. In our implementation, we chose to compensate (Fig. 5) only the dynamics of the 3 first axes, corresponding to the translation of the handle. Indeed, most of the dynamic effects are due to the most massive pieces of the HI, that are the basis and the two arms. It was also more simple to implement in a first approach.

The equations of motion of the simplified kinematical chain (Fig. 4) have been obtained thanks to the Lagrange Formalism of the principle of virtual power. Knowing the expression of the kinetic energy $T\left(E / R_{0}\right)$, the torques to be developed by the actuators to make the HI move can be expressed as follows:

$$
\tau_{\text {comp }}^{*}=\frac{d}{d t} \frac{\partial T\left(E / R_{0}\right)}{\partial \dot{q}}-\frac{\partial T\left(E / R_{0}\right)}{\partial q}+\mu \dot{\mathbf{q}}_{v}
$$

With $\tau_{\text {comp }}^{*}=\left[\tau_{\text {comp }}^{*} \tau_{\text {comp } p_{2}}^{*} \tau_{\text {comp }}^{*}\right]^{t}$ the vector of compensatory torques, $\mu=\left[\begin{array}{lll}\mu_{1} & \mu_{2} & \mu_{3}\end{array}\right]^{t}$ the vector of viscous friction coefficients and $\dot{\mathbf{q}}_{v}=$ 


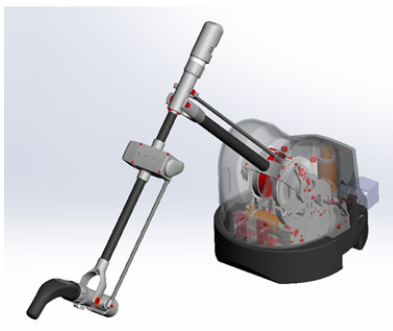

(a)

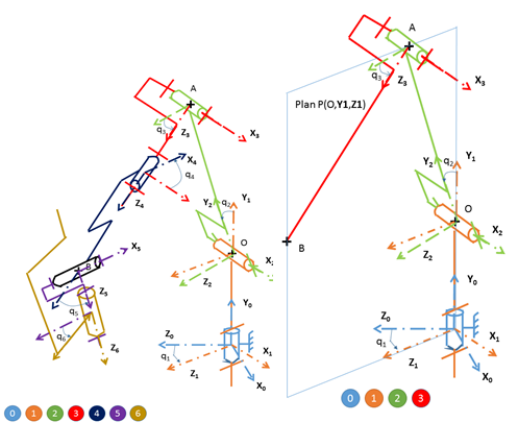

(b)

(c)

Fig. 4. Kinematical model of the HI. (a) Digital Mock-Up (b) Complete kinematical model (c) Simplified kinematical model (positioning the handle).

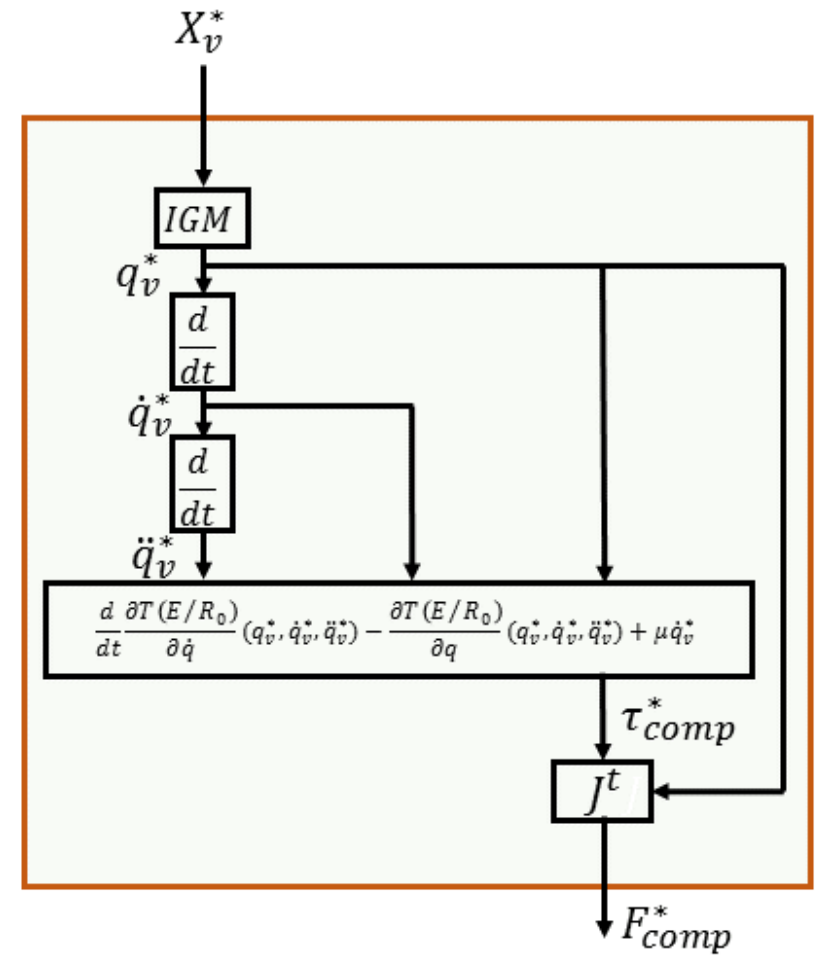

Fig. 5. Compensation model of the HI dynamics.

$\left[\begin{array}{lll}q_{v_{1}} & q_{v_{2}} & q_{v_{3}}\end{array}\right]^{t}$ the virtual joint velocities. 
$T\left(E / R_{0}\right)$ depends on mass an inertia properties of the bodies constituting the HI. In our case, we obtained these parameters from the digital mock-up. However, no data is available to determine the viscous friction coefficients associated to each axis. Therefore, we developed an experimental protocol to assess the strategy to be applied to generate the compensatory torques due to friction effects.

Passivity controller In addition to the control scheme presented above, we implemented a passivity controller (Fig. 6) as presented in [1719]. As it has been explained in the related work, this controller observes if the HI is dissipating energy, and modifies the force issued from the simulation is this is not the case to ensure the interface passivity. This algorithm is a safe guard, usually active for high impedance interactions (e.g. shocks, walls,...), since it modifies the force feedback and reduces transparency in consequence. $\alpha$ as seen in Fig. 6 is a coefficient applied to virtual power between the simulation and the HI, in order to adapt this power to satisfy the passivity condition and forces the simulation not to generate energy in the HI.

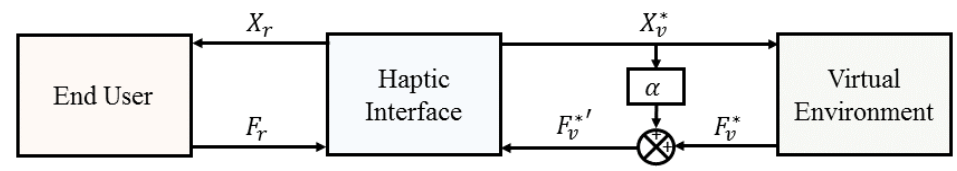

Fig. 6. Passivity controller added to the main control scheme. The controller was developed as a safe guard, and is normally not active during low mass object manipulations.

\subsection{Subjective assessment of friction compensation}

Since no data was available for the viscous friction coefficients $\mu$, we proposed to assess the effect of arbitrary compensations $\mu$ for the 3 first axes of the HI through a subjective evaluation of the fidelity of the interaction by a pool of subjects. Ten subjects participated to the experimentation. They were novices in haptic devices, on a scale of 1 to 7 they estimated to have an experience of $2.9 \pm 1.7$, and none of them declared pathologies of the arm in the last 6 months prior to the experiment.

After signing a consent form, each subject was asked to perform real and virtual tasks, as illustrated in Fig. 7, alternatively to test different friction compensations applied to the 3 first axes of the HI. The task consisted in moving a mass of $0.47 \mathrm{~kg}$ from an initial target to a final one through a passage above a small cylinder of $8 \mathrm{~cm}$. Targets were represented by a square of the size of the convex envelope of the object and a white circle represented the point to reach with the bottom of the handle of the haptic device. In order to minimize bias due to the difference of handling between the real and the virtual setup, the handle of the real object was 

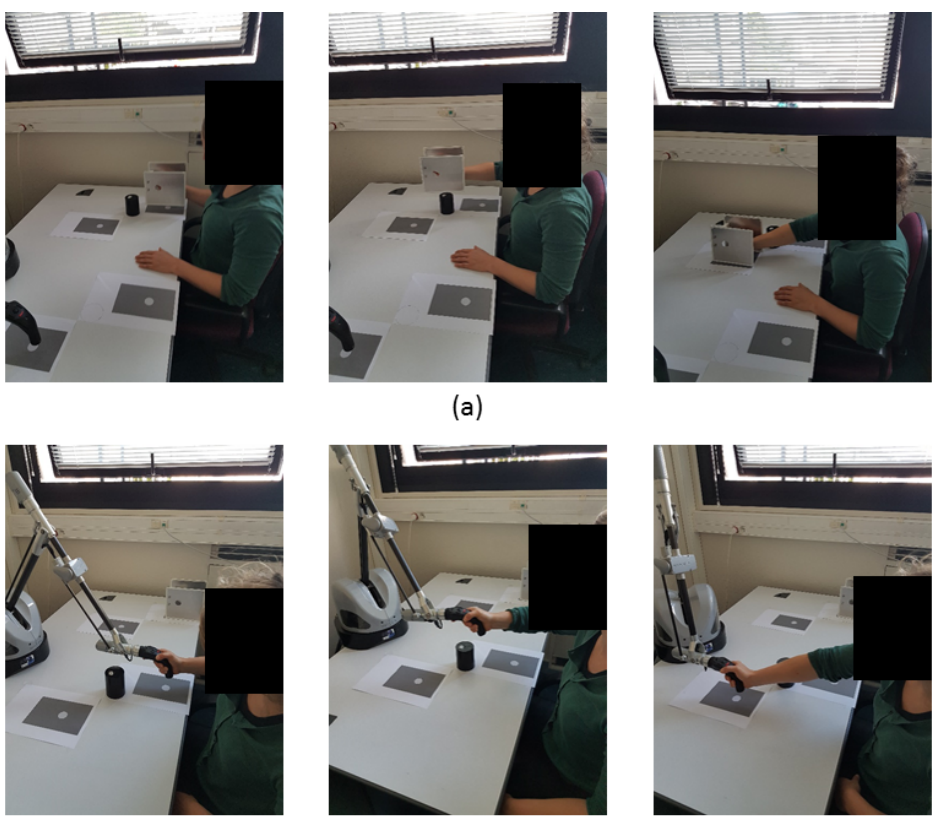

(a)
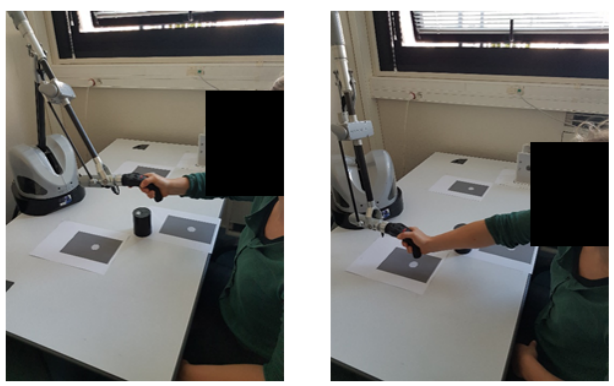

(b)

Fig. 7. Real (a) and virtual (b) setups used to assess the friction compensations applied to the haptic device. The subject has to move an object from one target to another with a passage above a small cylinder (height $=8 \mathrm{~cm}$ ).

designed and 3D printed to be a replica of the haptic device's one, and the object was balanced to avoid any torque interference.

The task was repeated 16 times in virtual and 16 times in real. After each trial in real, the subject tried in virtual and was asked to answer two questions on a Likert scale:

- Realism: Did you find the task close to the real one (1 not at all, 7 absolutely)?

- Perceived load: How do you judge the quantity of efforts to deploy in virtual with regard to the real setup (1 much less efforts, 7 much more efforts, 4 same efforts)?

The 16 conditions in virtual corresponded to the combination of friction compensation on the 3 first axes, repeated two times. The value of the fiction compensation was deducted from a previous experiment and was fixed to a unique value per axis to simplify the protocol. These have been set to $\mu_{1}=\mu_{2}=\mu_{3}=3 \mathrm{~kg} . \mathrm{s}^{-1}$. The 8 conditions repeated 2 times are summarized in Table 1.

Forces sent to the HI were logged during the experimentation to evaluate the ratio between the compensation forces sent to the HI and the forces arising from the rigid body motion.

Results of the questionnaire were statistically processed thanks to a 3way repeated measures ANOVA, with the friction coefficients as inde- 
Table 1. Task conditions in virtual.

\begin{tabular}{lc}
\hline Condition Friction compensation value per axis \\
\hline Condition 1 & $0-0-0$ \\
Condition 2 & $\mu_{1}-0-0$ \\
Condition 3 & $0-\mu_{2}-0$ \\
Condition 4 & $0-0-\mu_{3}$ \\
Condition 5 & $\mu_{1}-\mu_{2}-0$ \\
Condition 6 & $\mu_{1}-0-\mu_{3}$ \\
Condition 7 & $0-\mu_{2}-\mu_{3}$ \\
Condition 8 & $\mu_{1}-\mu_{2}-\mu_{3}$ \\
\hline
\end{tabular}

Table 2. Mean distances between HI control effort and its components. Significant effects are in bold. The closer the mean distance is to zero, the more the component is important in the HI control effort.

\begin{tabular}{lccc}
\hline & X-Axis & Y-Axis & Z-Axis \\
\hline Damping compensation & $\mathbf{0 . 2 6 7 2} \mathbf{N}$ & $\mathbf{0 . 1 9 7 4} \mathbf{N}$ & $4.5355 \mathrm{~N}$ \\
Inertia compensation & $0.3558 \mathrm{~N}$ & $0.6960 \mathrm{~N}$ & $4.5983 \mathrm{~N}$ \\
Force on the virtual object & $0.4429 \mathrm{~N}$ & $0.7314 \mathrm{~N}$ & $\mathbf{0 . 6 3 2 2} \mathbf{~ N}$ \\
\hline
\end{tabular}

pendent variables, the perceived load and the realism as dependent variables (level of confidence $\mathrm{p}<0.05$ ). Significant effects were investigated through interaction graphs and bar graphs.

\section{Results and Discussion}

Figure 8 shows the evolution of the HI control effort $F_{v}^{*}$ and its components during a task with the condition 8 (see Table 1). The compensation force is divided into damping and inertia compensations. Table 2 shows the mean distances between HI control force and its components for the same condition. Except on the Z-axis, the damping compensation are the most important component of the HI control force and the inertia compensation are the second one. The force applied on the virtual object is only predominant on the Z-axis because of the weight of the object. Therefore, the task chosen to illustrate the model based compensation method was relevant, since the compensation forces were of significant amplitude with regard to the simulation forces.

Table 3 shows the results of the 3-way repeated measures ANOVA. Since the data distribution was not checked, only significant effects were inves- 

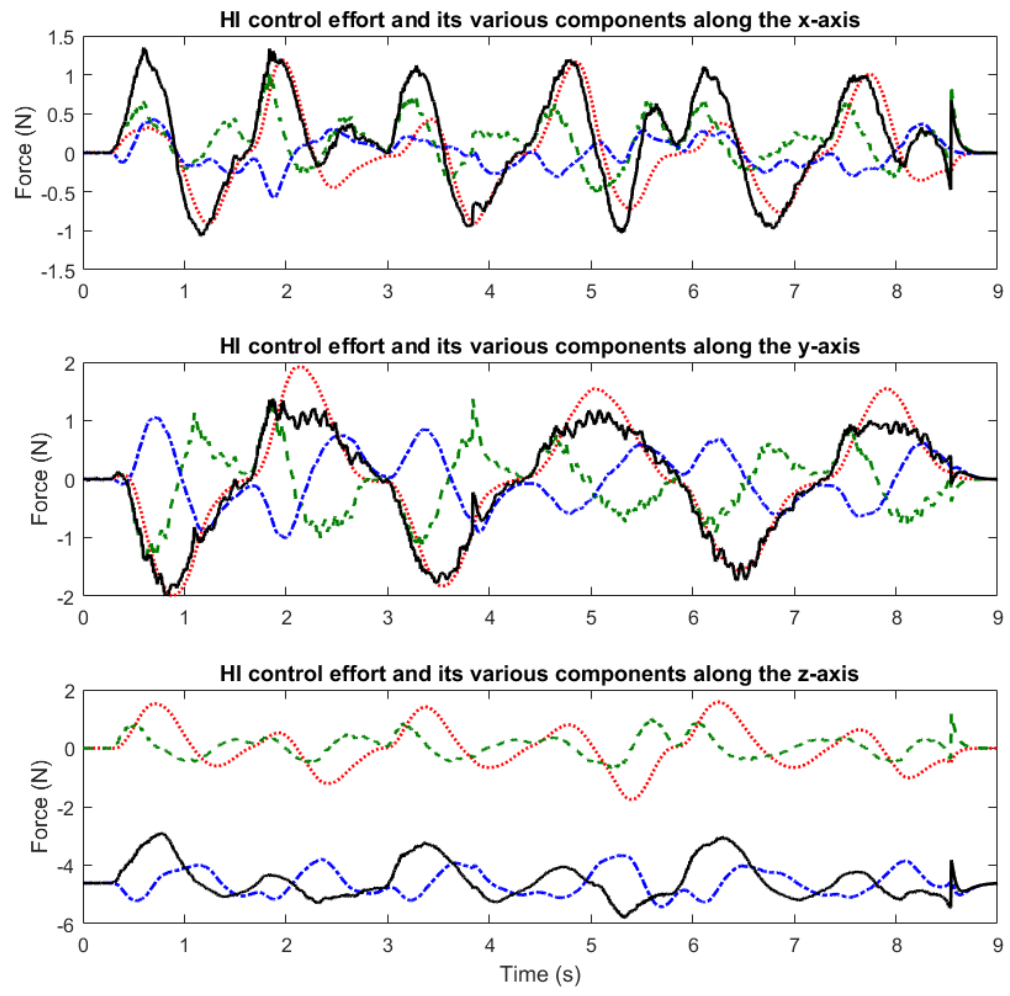

Fig. 8. HI control effort and its various components along the three axis. Red doted line represents the damping compensation, green dashed line represents the inertia compensation, blue dash-dot line represents the effort applied on the virtual object and the black line represents the HI control effort.

tigated. Results revealed a significant effect of the first axis compensation on the perceived load, and a significant interaction between the first and second axes compensation for the realism. These results were investigated through the plots presented in Fig. 9.

Figure 9 demonstrates the interest of the friction compensation on the realism for the second axis. The presence of the friction compensation on the first axis decreases slightly the realism in interaction with the second axis but this compensation is necessary since it enhances significantly the perceived load ( Fig. 9, the value is closer to the median value 4 with the $\mu_{1}$ compensation active). In other words, the compensation of the friction on the two first axes participated to enhance the transparency of the haptic interface, since it helped the user to better perceive the load and since it enhanced the realism of the task. 
Table 3. Repeated measures within subject ANOVA on Realism and Perceived Load. Significant effects are in bold and followed by a *

\begin{tabular}{|c|c|c|c|c|c|c|c|c|c|c|c|c|c|c|}
\hline \multirow{3}{*}{$\begin{array}{l}\text { Dependent } \\
\text { variables }\end{array}$} & \multirow{2}{*}{\multicolumn{2}{|c|}{$\mu_{1}$}} & \multirow{2}{*}{\multicolumn{2}{|c|}{$\mu_{2}$}} & \multirow{2}{*}{\multicolumn{2}{|c|}{$\mu_{3}$}} & \multicolumn{2}{|c|}{ Factors } & \multirow{3}{*}{$\begin{array}{c}\mu_{1} \times \\
\mathrm{F}\end{array}$} & \multirow[b]{2}{*}{$\mathbf{x} \mu_{3}$} & \multirow{3}{*}{$\begin{array}{l}\mu_{2} \\
\mathrm{~F}\end{array}$} & \multirow{3}{*}{$\begin{array}{c}\mathbf{x} \mu_{3} \\
\mathrm{p}\end{array}$} & \multirow{3}{*}{$\begin{array}{c}\mu_{1} \mathrm{x} \\
\mathrm{F}\end{array}$} & \multirow{3}{*}{$\begin{array}{c}\mu_{2} \times \mu_{3} \\
\mathrm{p}\end{array}$} \\
\hline & & & & & & & $\mu_{1} \mathrm{x}$ & $\mu_{2}$ & & & & & & \\
\hline & $\mathrm{F}$ & $\mathrm{p}$ & $\mathrm{F}$ & $\mathrm{p}$ & $\mathrm{F}$ & $\mathrm{p}$ & $\mathrm{F}$ & $\mathrm{p}$ & & $\mathrm{p}$ & & & & \\
\hline Realism & 1.14 & 0.31 & $5.25 *$ & 0.048 & 3.17 & 0.11 & $5.38^{*}$ & 0.046 & 0.015 & 50.90 & 3.83 & 0.082 & 0.14 & 0.72 \\
\hline Perceived Load & $8.86^{*}$ & 0.016 & 0.008 & 0.936 & 1.31 & 0.28 & 0.054 & 0.82 & 0.048 & 80.83 & 0.31 & 0.59 & 1.98 & 0.19 \\
\hline
\end{tabular}

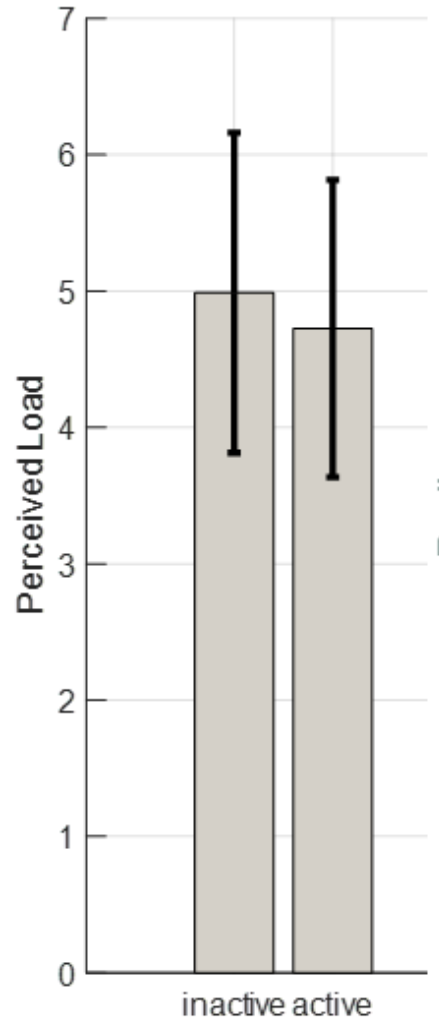

$\mu_{1}$

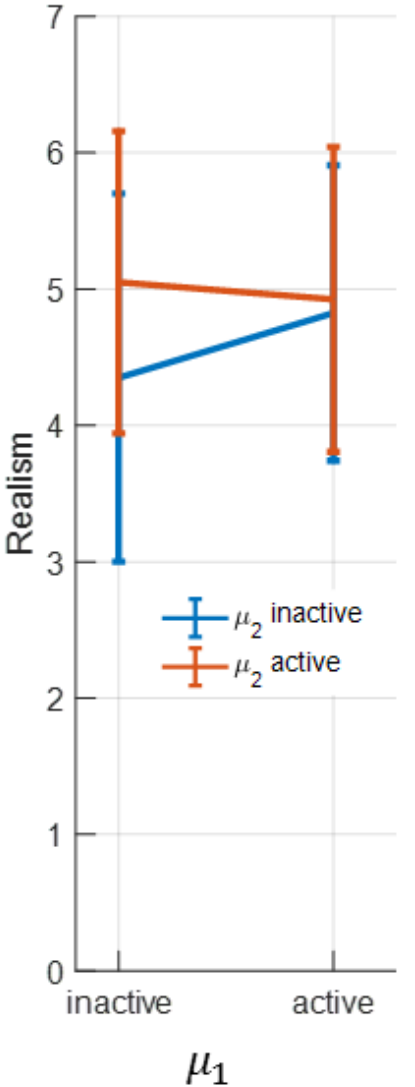

Fig. 9. Main effect of the friction coefficient $\mu_{1}$ on the perceived load and interaction effect of the friction coefficients $\mu_{1}$ and $\mu_{2}$ on the realism.

As a limit, the pool of subjects was quite limited and these results may have to be validated on a larger population. Moreover, the interest of the 
compensation itself (with or without the compensation algorithm) was not evaluated and it is an additional work that we need to pursue.

However, these results highlight a real interest of such compensatory process in order to increase the biomechanical fidelity of such a task realized in a virtual environment. Indeed, in an ergonomic setup, the user has to feel the task to be real, and he has to execute it naturally (in a comparative manner as he would have done in real) $[4,5]$. In the current work, we demonstrated that he would feel the task more realistically with a compensation, however we did not evaluate if the task was biomechanically done the same way as for real. To do so, it is necessary to evaluate objectively biomechanical quantities such as postures, joint angles or forces and compare them with the real task. Moreover, the comparison between what the user feels and what he actually does is of importance since it defines how transferable are the results from the virtual setup to the real world [6,7]. Indeed, the correlation between subjective ratings of discomfort and objective measurement such as postural rating and load capacity rating has been investigated in the past. For example, in [30], the authors showed a high correlation between subjective and objective indicators of discomfort. This means that a high level of subjective discomfort is generally associated with high scores in objective metrics. A virtual setup dedicated to ergonomics must exhibit the same behavior. However, due to cognitive differences and altered motor control strategies, this property is not always verified $[31,4,6]$.

Therefore, in a near future, we aim at evaluating the interest of such a compensation method in an immersive environment in comparison with a real setup, by measuring biomechanical quantities in virtual and real environments. The method will still be tested on low mass object manipulations, since this is an illustrative case of the interest of such compensation methods. Such developments can be made for several different tasks and may result in the development of alternative and relevant control strategies for haptic interfaces for ergonomic applications of virtual reality.

\section{Conclusion}

The current paper aimed at presenting a model based compensation command law enhancing the transparency and stability of a 6-dofs haptic device to simulate low mass objects manipulations. An experimental approach has been proposed to assess the effects of viscous friction coefficients compensation on the 3 first axes of the haptic arm. Results showed that the compensation of the friction on the first axis helped the users to better perceive the load to carry. The compensation of the friction on the second axis enhanced the sensation of the user to manipulate a real object during the task. The compensation of the friction on the third axis did not had any influence on the results. Therefore we consider using friction compensation of the two first axes in our next experiments to enhance the experience of the users.

The paper contribution lies in the fact that such model based compensation methods have not been extended to 6-dofs haptic interfaces as 
proposed here, and in the experimental approach used to assess the effect of the friction compensation.

Such results have a real interest to develop biomechanically realistic virtual setups, usable for ergonomic assessments of workstations. To make it possible, it will be necessary to assess the influence of the compensation method on the biomechanical fidelity of virtual environments.

\section{References}

1. Nguyen, H., Pontonnier, C., Hilt, S., Duval, T., Dumont, G.: Vrbased operating modes and metaphors for collaborative ergonomic design of industrial workstations. Journal on Multimodal User Interfaces 11(1) (2017) 97-111

2. Mavrikios, D., Karabatsou, V., Pappas, M., Chryssolouris, G.: An efficient approach to human motion modeling for the verification of human-centric product design and manufacturing in virtual environments. Robotics and Computer-Integrated Manufacturing 23(5) (2007) 533-543

3. Pappas, M., Karabatsou, V., Mavrikios, D., Chryssolouris, G.: Ergonomic evaluation of virtual assembly tasks. In: Digital enterprise technology. Springer (2007) 511-518

4. Pontonnier, C., Samani, A., Badawi, M., Madeleine, P., Dumont, G.: Assessing the ability of a vr-based assembly task simulation to evaluate physicalrisk factors. IEEE transactions on visualization and computer graphics 20(5) (2014) 664-674

5. Samani, A., Pontonnier, C., Dumont, G., Madeleine, P.: Kinematic synergy in a real and a virtual simulated assembly task. In: 19th Triennal Congress of the International Ergonomics Association (IEA2015). (2015)

6. Samani, A., Pontonnier, C., Dumont, G., Madeleine, P.: Shoulder kinematics and spatial pattern of trapezius electromyographic activity in real and virtual environments. PloS one 10(3) (2015) e0116211

7. Pontonnier, C., Dumont, G., Samani, A., Madeleine, P., Badawi, M.: Designing and evaluating a workstation in real and virtual environment: toward virtual reality based ergonomic design sessions. Journal on Multimodal User Interfaces 8(2) (2014) 199-208

8. Sagardia, M., Hulin, T.: Multimodal evaluation of the differences between real and virtual assemblies. IEEE Transactions on Haptics $\mathbf{P P}(99)$ (2017) 1-1

9. Baser, O., Gurocak, H., Konukseven, E.I.: Hybrid control algorithm to improve both stable impedance range and transparency in haptic devices. Mechatronics 23(1) (2013) 121 - 134

10. Bernstein, N.L., Lawrence, D.A., Pao, L.Y.: Friction modeling and compensation for haptic interfaces. In: First Joint Eurohaptics Conference and Symposium on Haptic Interfaces for Virtual Environment and Teleoperator Systems. World Haptics Conference. (March 2005) 290-295

11. Lyapunov, A.M.: The general problem of the stability of motion. International Journal of Control 55(3) (1992) 531-534 
12. Dang, Q.V.: Conception et commande d'une interface haptique à retour d'effort pour la CAO. PhD thesis, Université de Valenciennes et du Hainaut-Cambresis (2013)

13. Colgate, J.E., Schenkel, G.: Passivity of a class of sampled-data systems: application to haptic interfaces. In: American Control Conference, 1994. Volume 3. (June 1994) 3236-3240 vol.3

14. Colgate, J.E., Brown, J.M.: Factors affecting the z-width of a haptic display. In: Robotics and Automation, 1994. Proceedings., 1994 IEEE International Conference on, IEEE (1994) 3205-3210

15. Mehling, J.S., Colgate, J.E., Peshkin, M.A.: Increasing the impedance range of a haptic display by adding electrical damping. In: Eurohaptics Conference, 2005 and Symposium on Haptic Interfaces for Virtual Environment and Teleoperator Systems, 2005. World Haptics 2005. First Joint, IEEE (2005) 257-262

16. Weir, D.W., Colgate, J.E., Peshkin, M.A.: Measuring and increasing z-width with active electrical damping. In: Haptic interfaces for virtual environment and teleoperator systems, 2008. Haptics 2008. Symposium on, IEEE (2008) 169-175

17. Hannaford, B., Ryu, J.H.: Time-domain passivity control of haptic interfaces. IEEE Transactions on Robotics and Automation 18(1) (2002) $1-10$

18. Ryu, J.H., Kim, Y.S., Hannaford, B.: Sampled-and continuous-time passivity and stability of virtual environments. IEEE Transactions on Robotics 20(4) (2004) 772-776

19. Ryu, J.H., Preusche, C., Hannaford, B., Hirzinger, G.: Time domain passivity control with reference energy following. IEEE Transactions on Control Systems Technology 13(5) (2005) 737-742

20. Lim, Y.A., Ahn, H.S., Ryu, J.: Analogue input shaper for haptic interfaces. IET control theory \& applications 3(12) (2009) 15531564

21. Lee, K., Lee, D.Y.: Adjusting output-limiter for stable haptic rendering in virtual environments. IEEE Transactions on Control Systems Technology 17(4) (2009) 768-779

22. Franken, M., Stramigioli, S., Reilink, R., Secchi, C., Macchelli, A.: Bridging the gap between passivity and transparency, Robotics Science and Systems (2009)

23. Franken, M., Stramigioli, S., Misra, S., Secchi, C., Macchelli, A.: Bilateral telemanipulation with time delays: A two-layer approach combining passivity and transparency. IEEE Transactions on Robotics 27(4) (2011) 741-756

24. McJunkin, S.T.: Transparency improvement for haptic interfaces. $\mathrm{PhD}$ thesis, Rice University (2007)

25. Lee, H.K., Shin, M.H., Chung, M.J.: Adaptive controller of masterslave systems for transparent teleoperation. In: Advanced Robotics, 1997. ICAR'97. Proceedings., 8th International Conference on, IEEE (1997) 1021-1026

26. Carignan, C.R., Cleary, K.R.: Closed-loop force control for haptic simulation of virtual environments. The electronic journal of haptics research (2000) 
27. Frisoli, A., Sotgiu, E., Avizzano, C., Checcacci, D., Bergamasco, M.: Force-based impedance control of a haptic master system for teleoperation. Sensor Review 24(1) (2004) 42-50

28. Baser, O., Konukseven, E.I.: Utilization of motor current based torque feedback to improve the transparency of haptic interfaces. Mechanism and Machine Theory 52 (2012) 78-93

29. Baser, O., Konukseven, E.I., Gurocak, H.: Transparency improvement in haptic devices with a torque compensator using motor current. In: International Conference on Human Haptic Sensing and Touch Enabled Computer Applications, Springer (2012) 37-46

30. Kee, D., Lee, I.: Relationships between subjective and objective measures in assessing postural stresses. Applied Ergonomics 43(2) (2012) 277 - 282 Special Section on Product Comfort.

31. Hu, B., Ma, L., Zhang, W., Salvendy, G., Chablat, D., Bennis, F.: Predicting real-world ergonomic measurements by simulation in a virtual environment. International Journal of Industrial Ergonomics 41(1) (2011) $64-71$ 